\section{Sarcoidosis During Anti-Tumor Necrosis Factor- $\alpha$ Therapy: No Relapse After Rechallenge}

\section{To the Editor:}

Anti-tumor necrosis factor (TNF)- $\alpha$ agents such as infliximab, adalimum$a b$, and etanercept are more and more widely used. With this we see a growing number of case reports describing adverse events that occur during anti-TNF- $\alpha$ therapy. One adverse event that has been reported increasingly is the development of sarcoidosis ${ }^{1}$. This raises many questions. TNF- $\alpha$ plays an important role in the formation of granulomas. Based on this, specific anti-TNF- $\alpha$ agents are supposed to be effective in the treatment of granulomatous diseases such as Crohn's disease and sarcoidosis. Although current evidence supports the efficacy of infliximab in Crohn's disease, data regarding anti-TNF- $\alpha$ in sarcoidosis have been conflicting ${ }^{2,3}$.

We describe 2 cases in which sarcoidosis occurred during respectively adalimumab and etanercept treatment, both for rheumatoid arthritis. After cure/stabilization of the sarcoidosis both patients were rechallenged with the anti-TNF- $\alpha$ agent they took originally.

Case report 1 . The first patient was a 55-year-old woman with rheumatoid arthritis (RA) for 5 years. Eight months after treatment with adalimumab $40 \mathrm{mg}$ every 2 weeks, she developed erythema nodosum-like lesions on both knees as well as some dyspnea. A computed tomography (CT) scan of the thorax as well as a biopsy of the skin lesions (Figure 1) was performed. The CT scan of the thorax showed an extensive hilar and mediastinal lymphadenopathy. Bronchoalveolar lavage (BAL) showed a lymphocytosis with a CD4/CD8 ratio of 2/3. Cultures for routine bacteria and tuberculosis (TB) in sputum and BAL fluid were negative. No acid fast bacilli were found and polymerase chain reaction (PCR) for TB was negative as well. Patient had had a bacillus Calmette-Guerin vaccination so no purified protein derivative (PPD) test was performed. Serum angiotensin-converting enzyme (ACE) was normal (18-20 $\mu \mathrm{g} / \mathrm{l}$ on several occasions). Histological biopsies of the lung showed non-caseating granulomas. The skin biopsy

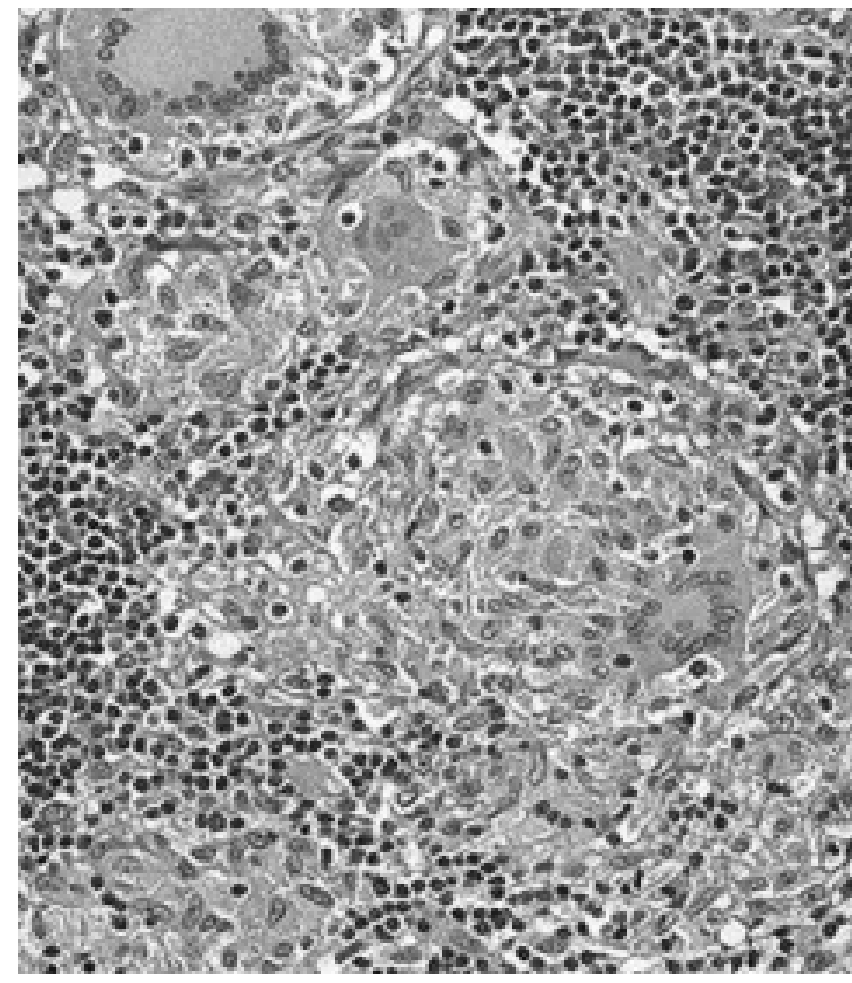

Figure 1. Skin biopsy of case 1 showing: Connective tissue stroma with epitheloid cell granulomas non-caseating and without signs of surrounding infiltration. HE stain 400x. showed non-caseating granulomas as well. At this stage the diagnosis of sarcoidosis was made and treatment with adalimumab was discontinued. In the months following, the patient recovered and the skin lesions as well as the lymphadenopathy disappeared.

Without the TNF- $\alpha$ therapy, however, her RA worsened. Because of the good effect on RA and disbelief that adalimumab was the causative agent for the sarcoidosis, adalumimab was reinitiated (8 months after discontinuation). It has been over 2 years since the adalimumab was restarted and to date the patient is still symptom-free.

Case report 2 . The second patient is a 51-year-old woman with RA for 14 years. Four years after the start of etanercept $50 \mathrm{mg}$ per week, she complained of cough, fever, a sore throat, and earache. At first it was thought to be a bacterial airway infection for which doxycyline and clarithromycin were prescribed. At the same time methotrexate and etanercept were discontinued. Her symptoms, however, did not improve and because of the discontinuation of the antirheumatic therapy, arthritis flared up. At the same time she developed a swollen parotid gland, as well as a cervical lymphadenopathy. Mumps was excluded by negative serology. The cough and lymphadenopathy persisted. CT thorax showed a hilar lymphadenopathy (Figure 2). Bronchoscopy showed no abnormalities. A BAL showed a lymphocytosis with a CD4/CD8 ratio of 12. Serum ACE was normal (5-21 $\mu \mathrm{g} / 1$ on several occasions). A histological lung biopsy and a biopsy of a supraclavicular lymph node both show non-caseating granulomas. Cultures for routine bacteria and TB in sputum and BAL fluid were negative. No acid fast bacilli were found and PCR for TB was negative as well. PPD test was not performed. At this stage a diagnosis of sarcoidosis was made.

Because of a flare of the RA, prednisone $10 \mathrm{mg}$ daily was initiated and under this therapy the parotid gland swelling disappeared as well as the cough. Hilar lymphadenopathy stabilized. When the patient was stable for 1 year the etanercept was reinitiated (17 mos after discontinuation) to optimize RA treatment. At present, the prednisone is stopped and she has been taking etanercept for 18 months without any indications of progression of the sarcoidosis.

Discussion. In both patients there was evidence for a sarcoidosis-like granulomatous disorder during anti-TNF- $\alpha$ therapy. Tuberculosis was ruled out. In the literature numerous cases are published about this subject ${ }^{1}$. In most cases no underlying infection could be proven. Some authors suggest that the anti-TNF- $\alpha$ agent might be the cause. Since TNF- $\alpha$ is important in granuloma formation, this at first seems unlikely. On the other hand, we see a higher incidence in granulomatous infections in patients receiving anti-TNF- $\alpha$ therapy. Cases of sarcoidosis during anti-TNF- $\alpha$ therapy described so far are mainly during etanercept treatment. This suggests a mechanism other than TNF- $\alpha$ suppression as the underlying cause. It has been suggested that anti-TNF- $\alpha$ therapy causes variation in cytokine balance that may favor the development of aseptic non-caseating granulomatous disease. However, the fact that there was no relapse after rechallenge with the original anti-TNF- $\alpha$ agent in the 2 case reports described here, makes a direct causative relation between the granuloma formation and the anti-TNF- $\alpha$ therapy unlikely. In our opinion, the best possible explanation is that the anti-TNF- $\alpha$ therapy created a condition in which an underlying infection, that we have not been able to prove, has caused the granulomatous disease we have indicated as sarcoidosis.

DEBORAH van der STOEP, MD; GERT-JAN BRAUNSTAHL, MD, PhD; JENDE van ZEBEN, MD, PhD; JACQUES WOUTERS, MD, PhD, Sint Franciscus Gasthuis - Rheumatology and Pulmonogy, Kleiweg 500, Rotterdam 3045 PM, the Netherlands. Address correspondence to D. van der Stoep. E-mail: dfvanderstoep@gmail.com

\section{REFERENCES}

1. Toussirot E, Pertuiset R, Kantelip B, Wendling D. Sarcoidosis occurring during anti-TNF-a treatment for inflammatory rheumatic diseases: report of two cases. Clin Exp Rheumatol 2008;26:471-5.

2. Baughman RP, Drent M, Kavuru M, Judson MA, Costabel U, du Bois R, et al. Infliximab therapy in patients with chronic 


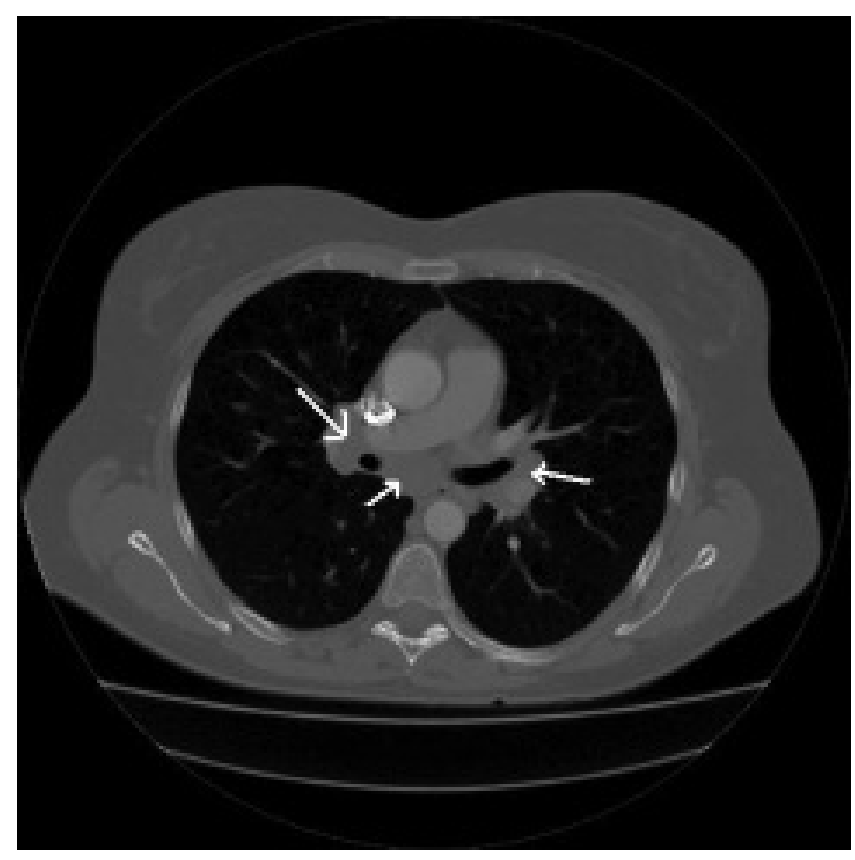

sarcoidosis and pulmonary involvement. Am J Respir Crit Care Med 2006;174:795-802.

3. Utz JP, Limper AH, Kalra S, Specks U, Scott JP, Vuk-Pavlovic Z, et al. Etanercept for the treatment of stage II and III progressive pulmonary sarcoidosis. Chest 2003;124:177-85.

J Rheumatol 2009;36:12; doi.10.3899/jrheum.090307

Figure 2. CT scan of the thorax of case 2 showing hilar lymphadenopathy. 